\title{
Impact of internal bremsstrahlung on the detection of $\gamma$-rays from neutralinos
}

\author{
M. Cannoni ${ }^{1}$, M. E. Gómez ${ }^{1}$, M. A. Sánchez-Conde ${ }^{2,3}$, F. Prada ${ }^{4}$ and O. Panella ${ }^{5}$ \\ 1 Departamento de Física Aplicada, Facultad de Ciencias Experimentales, Universidad de Huelva, 21071 Huelva, Spain \\ 2 Instituto de Astrofísica de Canarias (IAC), E-38200 La Laguna, Tenerife, Spain \\ 3 Departamento de Astrofísica, Universidad de La Laguna (ULL), E-38205 La Laguna, Tenerife, Spain \\ 4 Instituto de Astrofísica de Andalucía (CSIC), E-18008, Granada, Spain and \\ ${ }^{5}$ Istituto Nazionale di Fisica Nucleare, Sezione di Perugia, Via Alessandro Pascoli, 06129, Perugia, Italy
}

\begin{abstract}
We present a detailed study of the effect of internal bremsstrahlung photons in the context of the minimal supersymmetric standard models and their impact on $\gamma$-ray dark matter annihilation searches. We find that although this effect has to be included for the correct evaluation of fluxes of high energy photons from neutralino annihilation, its contribution is relevant only in models and at energies where the lines contribution is dominant over the secondary photons. Therefore, we find that the most optimistic supersymmetric scenarios for dark matter detection do not change significantly when including the internal bremsstrahlung. As an example, we review the $\gamma$-ray dark matter detection prospects of the Draco dwarf spheroidal galaxy for the MAGIC stereoscopic system and the CTA project. Though the flux of high energy photons is enhanced by an order of magnitude in some regions of the parameter space, the expected fluxes are still much below the sensitivity of the instruments.
\end{abstract}

PACS numbers: 95.35.+d, 95.55.Ka, 98.52.Wz, 12.60.Jv

The minimal supersymmetric (SUSY) extension of the standard model (MSSM) provides a natural candidate for dark matter (DM) in the form of a neutral, stable Majorana fermion, the lightest neutralino. At present, large efforts are being carried out to detect this SUSY DM by different methods, see [1] for reviews.

In the case of the current imaging atmospheric Cherenkov telescopes (IACTs), the searches are based on the detectability of $\gamma$-rays coming from the annihilation of the SUSY DM particles in the halo of galaxies [2]. Neutralinos annihilate at the one loop level into photons through the processes [3] $\chi \chi \rightarrow \gamma \gamma, \chi \chi \rightarrow Z \gamma$, with almost monochromatic outgoing photons of energies $E_{\gamma} \sim m_{\chi} E_{\gamma} \sim m_{\chi}-m_{Z}^{2} / 4 m_{\chi}$, respectively. Moreover, neutralino annihilation can produce a continuum spectrum of secondary photons from hadronization and decay of the annihilation products, mostly from neutral pion decay, which typically dominates over the number monochromatic $\gamma$ 's in a large portion of the parameter space. IACTs in operation like MAGIC, HESS, VERITAS [4] or satellites-based experiments like the Fermi satellite [5] play a very important role in this kind of DM searches. For these experiments, dwarf spheroidal (dSph) galaxies around the Milky Way represent a good alternative target option to e.g. the Galactic Center, already observed in $\gamma$-rays but with null DM detection so far [6]. Dsphs are DM dominated systems with inferred very high mass-to-light ratios, and most of them are expected to be free from any other astrophysical source that might contribute to a possible $\gamma$-ray signal. Therefore, the detection of $\gamma$-rays from them would probably imply a successful DM annihilation detection.

Some of the present authors calculated in Ref. 7] the expected $\gamma$-ray flux due to neutralino annihilation in the Draco dSph for a typical IACT above $100 \mathrm{GeV}$. Draco is located at $80 \mathrm{kpc}$ and is one of the dwarfs with more observational constraints, which have helped to better determine its DM density profile.

The MAGIC telescope has already observed Draco in $\gamma$-rays in the context of DM searches [8], but found no gamma signal above an energy threshold of $140 \mathrm{GeV}$. As a consequence, an upper limit for the flux ( $2 \sigma$ level) was set to be $1.1 \times 10^{-11} \mathrm{ph} \mathrm{cm}^{-2} \mathrm{~s}^{-1}$, assuming a power-law with spectral index -1.5 and a point-like source. This upper limit is $\mathcal{O}\left(10^{3}-10^{9}\right)$ above the values predicted by those SUSY models used in their analysis and therefore no constraints could be put on the parameter space. Also the Fermi collaboration has recently reported their upper limits for a possible $\gamma$-ray annihilation signal from Draco at lower energies [9], given that no significant gamma emission was detected above $100 \mathrm{MeV}$.

Recently, in Ref. [10], it was noted that the photons arising from internal bremsstrahlung (IB) in some regions of the parameter space can dominate the spectrum at energies near the neutralino mass. IB [10, 11] is commonly referred to the emission of additional photons in neutralino pair annihilation into charged final states, $\chi \chi \rightarrow X \bar{X} \gamma(X$ being a charged lepton, a quark, a $W$ boson or a charged Higgs) which is an unavoidable electromagnetic radiative correction. In the Feynman diagrams these photons can be attached to the external legs representing final state charged particles or to the propagator of the virtual charged particle exchanged by neutralinos: the latter diagrams are at the origin of the hard photon spectrum of IB.

The aim of this brief report is to quantitatively study the impact of the IB on the DM detection prospects for IACTs. As an example, we will revisit Draco updating the results obtained in Ref. [7], this time fully taking into account the IB contribution to the expected annihilation flux. We note that a study of Draco including IB was already done in Ref. [12], but only for a few benchmark 
points of the parameter space. Here, we will perform a wider exploration of the parameter space and will extract more general conclusions on the real importance of the IB for $\gamma$-ray DM searches.

The expected flux of photons with energy above the threshold of the telescope, $F\left(E_{\gamma}>E_{\mathrm{th}}\right)$, is given by the product of the so-called astrophysical factor $J(\Psi)$ times the particle physics factor, namely $\Phi_{P P}\left(E_{\gamma}>E_{\text {th }}\right)$. $J(\Psi)$ represents the integral of the square of the dark matter density $\rho_{D M}$ along the direction of observation $\Psi$ relative to the center of the DM halo, and depends on the PSF of the telescope. In the case of Draco, for instance, the authors in Ref. 7] used a cusp and a core DM density profiles, built from the latest stellar kinematic observations together with a rigorous method of removal of interloper stars. In the same work, they also stressed the important role of the PSF, which is directly related to the angular resolution of the IACT and becomes crucial for a correct interpretation of a possible gamma signal due to neutralino annihilation. However, for the sake of simplicity, we will use here the value of $J(\Psi)$ integrated over the whole spatial extent of the source as the value of the astrophysical factor. This value, that does not depend on the PSF any longer, can be well approximated by $\bar{J}=\frac{1}{4 \pi D^{2}} \int_{V} \rho_{D M}^{2}(r) d V$, with $D$ the distance from the Earth to the center of the DM halo and $r$ the galactocentric distance inside it. For Draco we take a value of $\bar{J}=3.7 \times 10^{17} \mathrm{Gev}^{2} \mathrm{~cm}^{-5}$, which was calculated using the cuspy DM density profile given in Ref. [7].

As for $\Phi_{P P}\left(E_{\gamma}>E_{\mathrm{th}}\right)$, which in the following we call $f_{S U S Y}$, it includes all the particle physics informations and is made up by the contribution of the continuum spectrum (secondaries and IB photons) and the monochromatic photons (lines):

$$
\begin{gathered}
f_{\text {susy }}=f_{\text {cont }}+f_{\text {lines }} \\
f_{\text {cont }}=\left(\sum_{f} B_{f} \int_{E_{t h}}^{m_{\chi}} \frac{d N_{\gamma}^{f}}{d E_{\gamma}} d E_{\gamma}\right) \frac{\left\langle\sigma_{\chi \chi} v\right\rangle}{2 m_{\chi}^{2}}=f_{\text {sec }}+f_{I B}, \\
f_{\text {lines }}=2 \frac{\left\langle\sigma_{\gamma \gamma} v\right\rangle}{2 m_{\chi}^{2}}+\frac{\left\langle\sigma_{Z \gamma} v\right\rangle}{2 m_{\chi}^{2}} .
\end{gathered}
$$

Here $d N_{\gamma}^{f} / d E_{\gamma}$ is the differential yield of photons per annihilation to the final state $f$ with branching ratio $B_{f}$. The factor in parenthesis is thus $n_{\gamma}\left(E_{\gamma}>E_{t h}\right)$, the total number of photons per annihilation with energy greater than the threshold energy, $\left\langle\sigma_{\chi \chi} v\right\rangle$ is the thermal averaged total neutralino annihilation cross, $\left\langle\sigma_{\gamma \gamma} v\right\rangle$ and $\left\langle\sigma_{Z \gamma} v\right\rangle$ the cross sections for annihilation into lines and $m_{\chi}$ the neutralino mass.

As in Ref. [7] we assume that the neutralino is the main component of the DM present in the Universe with abundance inside the cosmologically favored interval $0.09<\Omega_{\chi} h^{2}<0.13$ (the most recent WMAP [13] interval at $3 \sigma$ is $\left.0.094<\Omega_{D M} h^{2}<0.128\right)$. We further require that SUSY models satisfy the LEP bounds on Higgs and chargino masses, $m_{h}>114 \mathrm{GeV}$ and $m_{\chi^{+}}>103.5 \mathrm{GeV}$, and constraints from $b \rightarrow s \gamma$ as explained in Refs. [7, 15]. We first consider minimal supergravity (mSUGRA) models, where the soft terms of the MSSM are taken to be universal at the gauge unification scale $M_{G U T}$. The effective theory at energies below $M_{G U T}$ is thus determined by four universal parameters: the common scalar mass $m_{0}$, the gauginos mass $m_{1 / 2}$, the trilinear couplings $A_{0}$, and the ratio of the Higgs vacuum expectation values, $\tan \beta$. In addition, the minimization of the Higgs potential leaves undetermined the sign of the Higgs mass parameter $\mu$ that we take positive. For the numerical computation of IB effects we use DarkSusy 5.0.5 [14] which relies on ISASUGRA 7.78 for the renormalization group equation evolution of parameters.

The amount of photons coming from IB depends on the annihilation channels and therefore it depends on the nature of the neutralino in every particular SUSY model. We thus display in Fig. 1 the differential yield of photons for some representative points of the mSUGRA parameter space specified in Table \ where the distinct contributions to $f_{S U S Y}$ integrating the number of photons above $E_{t h}=100 \mathrm{GeV}$ can be read. Model A is in the stau coannihilation region of the mSUGRA parameter space: the mass of the lightest stau is $m_{\tilde{\tau}}=195$ $\mathrm{GeV}$ very close to $m_{\chi}=188 \mathrm{GeV}$. Neutralino pair annihilation in $\tau^{+} \tau^{-}$mediated by $t$-channel exchange of stau has the highest annihilation cross section thus IB is relevant. Here $f_{I B}$ is the dominant contribution being 10 and 4.4 times greater than $f_{\text {sec }}$ and $f_{\text {lines. }}$. Model $\mathrm{B}$ is in the funnel or resonances region: the mass of the CPodd neutral Higgs is $m_{A}=1211 \mathrm{GeV}$ while $m_{\chi}=598$ $\mathrm{GeV}$, thus $m_{A} \simeq 2 m_{\chi}^{0}$ and WMAP bounds are satisfied due to neutralino pair annihilation into fermion through $s$-channel exchange of heavy neutral Higgs bosons. In this case no photon line can be attached to the virtual particles and the IB yield is negligible. Model C is in the focus point or hyperbolic branch region. The mass of the lightest chargino is $m_{\chi^{ \pm}}=212 \mathrm{GeV}$, not much bigger than $m_{\chi}=163 \mathrm{GeV}$ and neutralino pairs annihilate into $W^{+} W^{-}$through $t$-channel chargino exchange. The IB yield is small because $m_{\chi}$ is not much greater than $m_{W}$ and photons energy has a cut off which corresponds to the kinematic endpoint $x=1-m_{W}{ }^{2} / m_{\chi}^{2} \sim 0.75$. Here $f_{\text {lines }}$ is bigger than $f_{\text {sec }}$ and $f_{I B}$. Model $\mathrm{D}$ is another example in the focus point region. The mass of the lightest chargino is $m_{\chi^{ \pm}}=954 \mathrm{GeV}$, almost degenerate with $m_{\chi}=918 \mathrm{GeV}$. Neutralino pairs annihilate into $W^{+} W^{-}$ through $t$-channel chargino exchange as in $\mathrm{C}$ but in this case $m_{\chi} \gg m_{W}$ thus IB photons contribution is more important and have endpoint at the neutralino mass: here $f_{S U S Y}$ is dominated by $f_{s e c}$. The point $\mathrm{E}$ is similar to point A but with even more pronounced IB contribution (here $\left.m_{h}=113 \mathrm{GeV}\right) . f_{I B}$ is a factor 50 and 5 greater than $f_{\text {sec }}$ and $f_{\text {lines }}$ respectively but the the total $f_{S U S Y}$ is the smaller one.

These ratios are quite general as can be seen in Fig. 2 where we present a larger scan on the parameter space of $f_{S U S Y}$ versus the threshold energy setting $A_{0}=0$ and two values of $\tan \beta, 10$ and 50 . In panel $(a)$ where points are in the stau coannihilation region we can appreciate the largest contribution of IB, as shown by the points A 
TABLE I. mSUGRA models used in Fig. 1 The values of $m_{0}, m_{1 / 2}, A_{0}, m_{\tilde{\chi}}$ are in GeV, the sign of $\mu$ is positive. The units of $\left\langle\sigma_{\chi \chi} v\right\rangle$ are $\mathrm{cm}^{3} \mathrm{~s}^{-1}$, those of the $f$ 's, defined in Eq. (11), are $\mathrm{GeV}^{-2} \mathrm{~cm}^{3} \mathrm{~s}^{-1}$.

\begin{tabular}{cccccccccccc}
\hline \hline & $\tan \beta$ & $m_{0}$ & $m_{1 / 2}$ & $A_{0}$ & $m_{\tilde{\chi}}$ & $\Omega_{\chi} h^{2}$ & $\left\langle\sigma_{\chi \chi} v\right\rangle \cdot 10^{29}$ & $f_{\text {sec }} \cdot 10^{32}$ & $f_{\text {lines }} \cdot 10^{32}$ & $f_{I B} \cdot 10^{32}$ & $f_{\text {SUSY }} \cdot 10^{32}$ \\
\hline $\mathrm{A}$ & 18 & 127 & 459 & -135 & 187.6 & 0.092 & 29 & 0.008 & 0.018 & 0.079 \\
$\mathrm{~B}$ & 52 & 982 & 1377 & 725 & 597.6 & 0.092 & 2600 & 0.72 & $10^{-5}$ & $10^{-5}$ \\
$\mathrm{C}$ & 17 & 2200 & 430 & 805 & 162.8 & 0.098 & 2225 & 0.04 & 0.06 & 0.02 \\
$\mathrm{D}$ & 51 & 8940 & 2218 & -4221 & 918.2 & 0.099 & 1203 & 0.3 & 0.003 & 0.017 & 0.12 \\
$\mathrm{E}$ & 5 & 110 & 530 & -600 & 218.4 & 0.1 & 11.2 & 0.0014 & 0.014 & 0.073 & 0.082 \\
\hline \hline
\end{tabular}

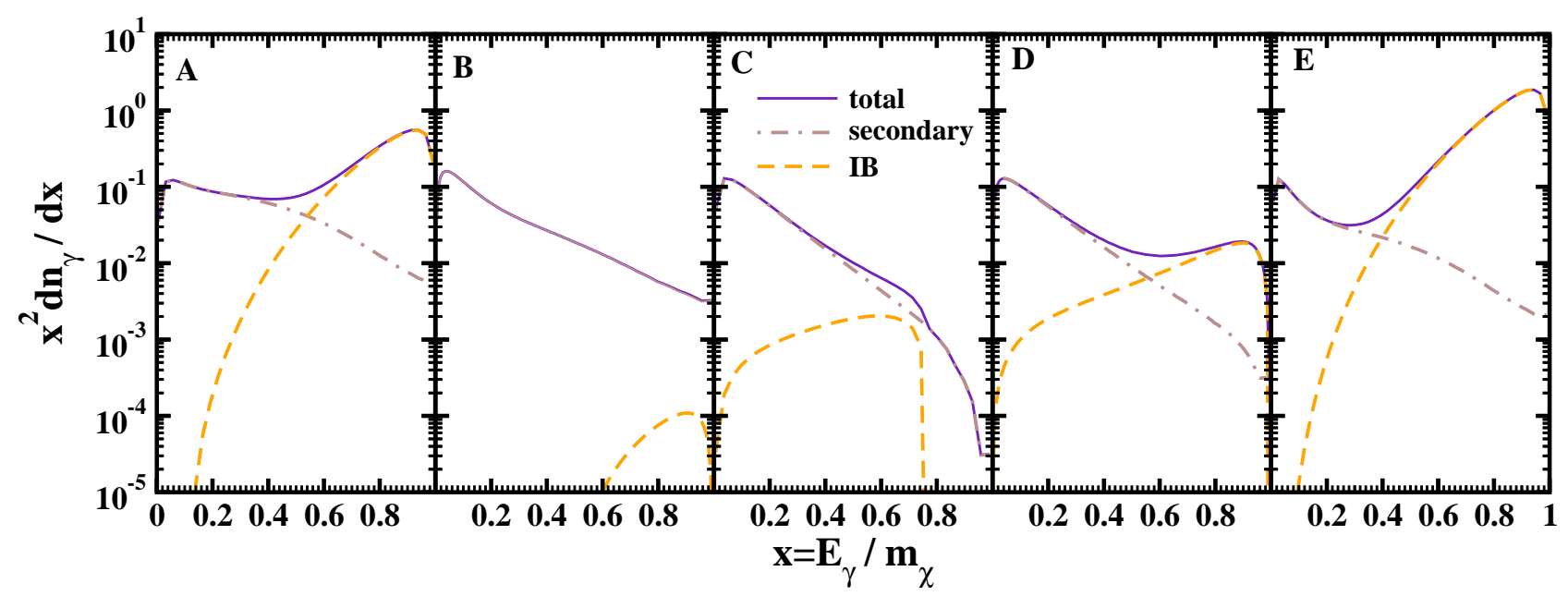

FIG. 1. Continuum $\gamma$-rays spectra for the models in Table 1 The solid lines indicate the total yield, the dot-dashed lines the contribution of secondary photons, while dashed lines correspond just to the IB contribution.

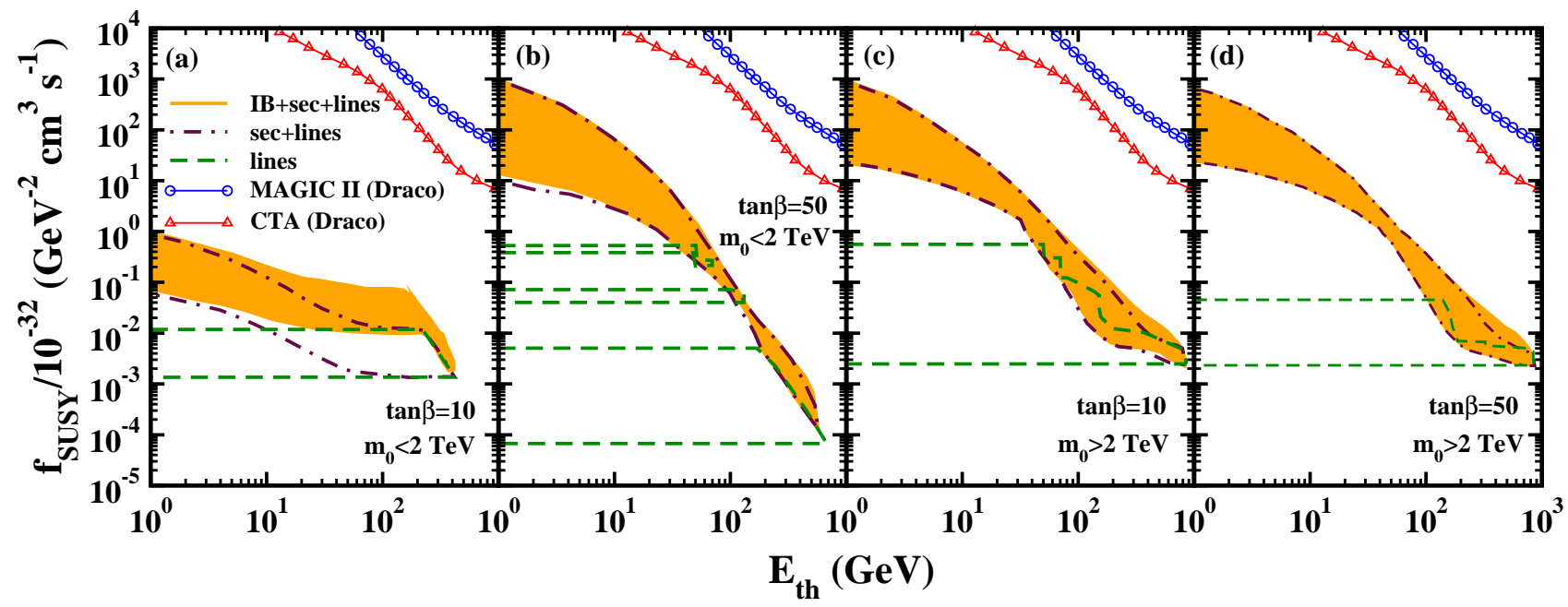

FIG. 2. The particle physics factor $f_{S U S Y}$, Eq. (1), versus $E_{t h}$, energy threshold of the detector. We set $A_{0}=0$ and $\mu$ positive while $m_{0}$ and $m_{1 / 2}$ have values such that the mSUGRA point predicts the neutralino relic density inside the WMAP bounds satisfying all the phenomenological constraints. Also plotted are the predicted sensitivity lines of MAGIC II and CTA for Draco corresponding to 50 hours of observation time and a $5 \sigma$ detection level.

and E. The absence of IB photons of the point B is evidenced by the panel $(b)$ where points are in the funnel region, while the panels $(c)$ and $(d)$ have points mostly in the hyperbolic branch and share properties with the points $\mathrm{C}$ and $\mathrm{D}$. We also plot in those panels the sensi- tivity lines for Draco of the MAGIC telescopes in stereoscopic mode [16] and of the CTA project [17] as given by Montecarlo simulations for 50 hours of observation time and a $5 \sigma$ detection level. We see that even including IB for a typical value of the threshold $E_{t h}=100 \mathrm{GeV}$, the 


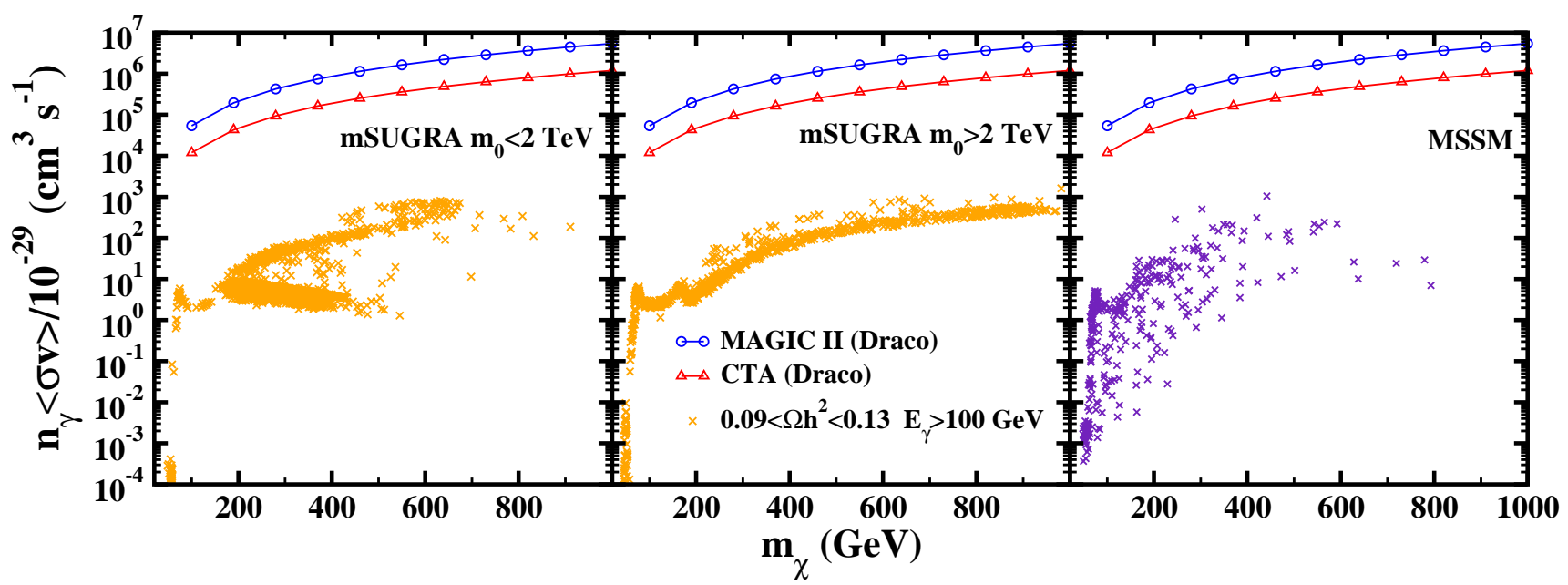

FIG. 3. Scatter plot of $n_{\gamma}\left(E_{\gamma}>100 \mathrm{GeV}\right) \times\left\langle\sigma_{\chi \chi} v\right\rangle$ versus $m_{\chi}$ for continuum photons in mSUGRA and general MSSM. Also plotted are the predicted sensitivity lines of MAGIC II and CTA for Draco assuming $E_{t h}=100 \mathrm{GeV}, 50$ hours of observation time and a $5 \sigma$ detection level.

theoretical predictions are at least three orders of magnitude below the detection limit.

We further perform a scan on more general parameter space both in mSUGRA and in a general MSSM with random soft terms at the electroweak scale (see Ref. 7] for details on the scanned parameter space). In Fig. 3 we plot the quantity $n_{\gamma}\left(E_{\gamma}>E_{t h}\right) \times\left\langle\sigma_{\chi \chi} v\right\rangle$ versus $m_{\chi}$ together with the MAGIC II and CTA sensitivity lines assuming $E_{t h}=100 \mathrm{GeV}$ : as above we see that a boost of at least three and two orders of magnitude respectively is needed to reach the detection line. We note, however, that the effect of substructures in the dwarf may enhance the annihilation flux importantly (see e.g. Ref. [18]).

In summary, we have reported a detailed study of the internal bremsstrahlung contribution to the expected $\gamma$ ray flux from neutralino annihilation. We have found that although this effect has to be included for evaluation of fluxes of high energy photons from neutralino annihilation, its contribution is relevant only in models and at energies where the lines contribution is dominant over the secondary photons. As a result, the most optimistic particle physics scenarios for DM detection (which typi- cally correspond to those where most of the flux is given by secondary photons) will not change substantially. On the other hand, being typically the IB yield at most an order of magnitude greater than the lines yield, the net increase on absolute flux is of the same order. As an example of the impact of the IB on previous works on DM search, we recalculated the DM detection prospects of the Draco dwarf galaxy for the MAGIC telescopes, updating a previous work by some of the authors. We find that though the effect can rise the flux by e.g. an order of magnitude at $100 \mathrm{GeV}$, the predicted fluxes are still at least three orders of magnitude below the sensitivity of the instrument both in mSUGRA SUSY scenario and in the general MSSM. The same is applicable to other IACTs, given their roughly similar sensitivities at those energies.

The authors acknowledges support from the project P07FQM02962 funded by "Junta de Andalucia", the Spanish MICINN-INFN(PG21) projects FPA200910773, FPA2008-04063-E and MULTIDARK project of Spanish MICINN Consolider-Ingenio 2010: CSD200900064 .
[1] G. Bertone, D. Hooper and J. Silk, Phys. Rept. 405, 279 (2005); C. Munoz, Int. J. Mod. Phys. A 19, 3093 (2004).

[2] Y. B. Zeldovich, A. A. Klypin, M. Y. Khlopov and V. M. Chechetkin, Sov. J. Nucl. Phys. 31, 664 (1980) [Yad. Fiz. 31, 1286 (1980)]; J. Silk and M. Srednicki, Phys. Rev. Lett. 53, 624 (1984).

[3] L. Bergstrom and P. Ullio, Nucl. Phys. B 504, 27 (1997); Z. Bern, P. Gondolo and M. Perelstein, Phys. Lett. B 411, 86 (1997); P. Ullio and L. Bergstrom, Phys. Rev. D 57, 1962 (1998).

[4] E. Lorenz [MAGIC Collaboration], New Astron. Rev. 48, 339 (2004); J. A. Hinton [The HESS Collaboration], New
Astron. Rev. 48, 331 (2004); T. C. Weekes et al., Astropart. Phys. 17, 221 (2002).

[5] N. Gehrels and P. Michelson, Astropart. Phys. 11, 277 (1999).

[6] F. Aharonian et al. [The HESS Collaboration], Astron. Astrophys. 425, L13 (2004); J. Albert et al. [MAGIC Collaboration], Astrophys. J. 638, L101 (2006); F. Aharonian et al. [H.E.S.S. Collaboration], Phys. Rev. Lett. 97, 221102 (2006) [Erratum-ibid. 97, 249901 (2006)].

[7] M. A. Sanchez-Conde, F. Prada, E. L. Lokas, M. E. Gomez, R. Wojtak and M. Moles, Phys. Rev. D 76, 123509 (2007). 
[8] J. Albert et al. [MAGIC Collaboration], Astrophys. J. 679, 428 (2008).

[9] A. A. Abdo et al., Astrophys. J. 712, 147 (2010).

[10] T. Bringmann, L. Bergstrom and J. Edsjo, JHEP 0801, 049 (2008).

[11] L. Bergstrom, Phys. Lett. B 225, 372 (1989); R. Flores, K. A. Olive and S. Rudaz, Phys. Lett. B 232, 377 (1989); L. Bergstrom, T. Bringmann, M. Eriksson and M. Gustafsson, Phys. Rev. Lett. 95, 241301 (2005); V. Barger, Y. Gao, W. Y. Keung and D. Marfatia, Phys. Rev. D 80, 063537 (2009).

[12] T. Bringmann, M. Doro and M. Fornasa, JCAP 0901, 016 (2009).

[13] D. Larson et al., arXiv:1001.4635 [astro-ph.CO].
[14] P. Gondolo, J. Edsjo, P. Ullio, L. Bergstrom, M. Schelke and E. A. Baltz, JCAP 0407, 008 (2004); F. E. Paige, S. D. Protopopescu, H. Baer and X. Tata, arXiv:hep-ph/0312045

[15] M. E. Gomez, T. Ibrahim, P. Nath and S. Skadhauge, Phys. Rev. D 72, 095008 (2005); ibid. 74, 015015 (2006); M. E. Gomez, G. Lazarides and C. Pallis, Nucl. Phys. B 638, 165 (2002), Phys. Rev. D 67, 097701 (2003); M. Cannoni and O. Panella, Phys. Rev. D 81, 036009 (2010).

[16] P. Colin et al. [MAGIC collaboration], arXiv:0907.0960.

[17] M. Doro [CTA collaboration], arXiv:0908.1410.

[18] L. Pieri, A. Pizzella, E. M. Corsini, E. D. Bonta' and F. Bertola, Astron. Astrophys. 496, 351 (2009). 